\title{
Field profiles for spherical conductive nanoparticles and metallic-shell/dielectric-core nano-composites
}

\author{
S. Schelm and G. B. Smith \\ Department of Applied Physics, University of Technology, Sydney, PO Box 123 Broadway, \\ NSW 2007, Sydney, Australia
}

\begin{abstract}
Profiles of the electric field strength $|\mathrm{E}|^{2} /\left|\mathbf{E}_{0}\right|^{2}$ for spherical metallic shells on a dielectric core are presented both inside the particle and outside. The dependence of the near-field strength and extent on shell thickness and total particle size is discussed qualitatively. Although the internal fields inside the shell and in the core are larger than for homogeneous particles, for not too thick shells, this does not translate into a stronger near-field away from the surface of the shell. The fields inside the shell, at the low energy resonance and close to it, are rotated by $\frac{\pi}{2}$ with respect to fields inside homogeneous particles, which means that the maximum field strengths in the shell are perpendicular to the incident polarisation. This follows from the fact that the low energy resonance for a shell is for the largest dipole moment of the whole system, which compensates the incident field. The largest moment is created when the same charges are collected at both interfaces (shell/medium and core/shell) along the incident polarisation. This creates regions of low field densities at the poles along the incident polarisation, because same charge fields repel each other. Following from that, the field lines are bunched up at the perpendicular poles, creating large field line densities and hence large fields at these points. The case for opposite charges across the interfaces creates the high energy, antisymmetric resonance.
\end{abstract}

Keywords: nanoparticles, core-shell particles, electric field profiles, optical properties

\section{INTRODUCTION}

Metallic shells with a diameter on the nanometre scale have attracted an increased interest over recent years. ${ }^{1-5}$ While the electromagnetic theory for these structures, as an extension of the original Mie theory for homogeneous particles, ${ }^{6}$ has been around for some time,,${ }^{7,8}$ the controlled manufacture of those structures became only possible some 40 years later. ${ }^{9,10}$ This and a broad range of possible applications, near infrared absorbers, SERS substrates, sensors and drug delivery, to name just a few, ${ }^{4,11-13}$ created a large research interest over the last couple of years. One of the interests is the improvement of the synthesis (almost exclusively chemically), ${ }^{2,14,15}$ with respect to homogeneity of the coating and reduced poly-dispersity, for example, while another is the theoretical and computational description. ${ }^{3,16,17}$ The computational side to the solution of Mie theory for homogeneous and coated particles has been discussed for some years ${ }^{18-20}$ and various codes are available, ${ }^{21}$ though there remain some unresolved issues which call for improvement. They have mainly to do with the stability in the calculation of the spherical harmonic functions. ${ }^{22}$

The main applications for programs dealing with coated spheres have been, up until some years ago, dielectricdielectric structures or spherical cavities, both of which express so called morphological dependent resonances ${ }^{23-25}$ (MDR) (also called whispering gallery modes) and structures of meteorological importance (e.g. a soot layer on a water sphere).$^{26}$ This is especially true for the internal fields, which only recently have been calculated for metallic shells. ${ }^{3,17 *}$

The reason for the strong optical properties for metallic nanostructures is the excitation of surface plasmons at the interface between the metal and the medium. In isolated particles these plasmons are called localised surface plasmons resonances (LSPR), whereas in thin films and percolated structures propagating plasmons can be excited which are called surface plasmon polaritons (SPP). As we only deal here with isolated particles the surface plasmons occurring are LSPRs.

Correspondence should be addressed to: stefan.schelm@uts.edu.au

*In Ref. 3 the field inside the actual shell is not calculated, which is due to the specific approximation used 


\section{THEORY}

Although the original theory by Mie describes only the properties of homogeneous spheres, ${ }^{6}$ it's extension to a coated sphere is straightforward and was done more than 50 years ago. ${ }^{7,8}$ The differences are mainly the extension of the boundary conditions to the second interface and the determination of the coefficients for the series expansion in the shell. Also the functional form of the coefficients in the core and of the scattered wave are more complex than in the case of a homogeneous sphere.

Other problems involved in the case of coated spheres and stratified, multilayered spheres are the precision and reliability of existing codes, which mainly depends on the calculation of Bessel functions with complex and/or large arguments and calculations for higher orders of these functions. This is actually still a field of active research and important improvements are still being produced. ${ }^{22}$ The system of a highly absorbing very thin layer on a larger (micrometer scale) particle is still difficult to calculate with current Mie codes. One of the main aims in the computational solution to Mie theory is to rephrase the equations for the expansion coefficients into forms which are better suited to computer calculations. Two approaches commonly used for this are logarithmic derivatives and ratios of the Riccati-Bessel functions. ${ }^{19,20}$

We used a code developed by $\mathrm{T}$. Kaiser et al. ${ }^{20}$ for which they rewrote the formulas for the expansion coefficients in a way which is more suitable for computations. The original code separated the response into the internal fields and the scattered fields and includes varies extensions for the scattered case (e.g. size distributions). The problem with the external part of the code is that it assumes the far-field approximations and is therefore not valid for calculations of the near-field response. As the internal code actually calculates the scattering coefficients we extended this part of the code, based on the algorithm from Bohren and Huffman. ${ }^{19}$

The external electric field is given by the following equation:

$$
\mathbf{E}=\sum_{n=1}^{\infty} E_{n}\left(i a_{n} \mathbf{N}_{e 1 n}^{(3)}-b_{n} \mathbf{M}_{o 1 n}^{(3)}\right) \text {, with } E_{n}=i^{n} E_{0} \frac{(2 n+1)}{n(n+1)},
$$

where $a_{n}$ and $b_{n}$ are the Mie scattering coefficients and $\mathbf{N}_{e 1 n}^{(3)}$ and $\mathbf{M}_{o 1 n}^{(3)}$ are the spherical harmonics for the electric field, created by the Hankel function of the first kind $h_{n}^{(1)}(\rho)$. In the case of the electric field inside the core of the particle, the coefficients and the generating function for the spherical harmonics $\mathbf{N}_{e 1 n}$ and $\mathbf{M}_{o 1 n}$ change. In the shell the coefficients not only change but there are two additional coefficients, because inside the shell both generating functions (for the core and the scattered one) are valid solutions. Separated into the contributions for the three variables in spherical coordinates $(r, \theta, \phi)$ we can write for the external field:

$$
\begin{aligned}
& \mathbf{E}_{r}=\frac{\cos \phi \sin \theta}{\rho} \sum_{n=1}^{\infty} i^{n+1}(2 n+1) a_{n} \pi_{n}(\cos \theta) h_{n}^{(1)}(\rho) \overrightarrow{\mathbf{e}}_{r} \\
& \mathbf{E}_{\theta}=\cos \phi \sum_{n=1}^{\infty} i^{n} E_{0} \frac{(2 n+1)}{n(n+1)}\left(i a_{n} \tau_{n}(\cos \theta) \frac{\left(\rho h_{n}^{(1)}(\rho)\right)^{\prime}}{\rho}-b_{n} \pi_{n}(\cos \theta) h_{n}^{(1)}(\rho)\right) \overrightarrow{\mathrm{e}}_{\theta} \\
& \mathbf{E}_{\phi}=-\sin \phi \sum_{n=1}^{\infty} i^{n} E_{0} \frac{(2 n+1)}{n(n+1)}\left(i a_{n} \pi_{n}(\cos \theta) \frac{\left(\rho h_{n}^{(1)}(\rho)\right)^{\prime}}{\rho}-b_{n} \tau_{n}(\cos \theta) h_{n}^{(1)}(\rho)\right) \overrightarrow{\mathbf{e}}_{\phi},
\end{aligned}
$$

where $\tau_{n}$ and $\pi_{n}$ are angle-dependent functions ${ }^{19}$ and $\rho=k r, k$ being the wave number in the medium and $r$ being the radial distance of the test point. The fields for the shell and the core follow similar expressions. The full expressions for the internal fields and the expansion coefficients can be found in Ref. 20.

The properties of homogeneous spheres and metallic shells with respect to the LSPR position and near-field properties are different. A not too thick metallic shell will show a spectral red-shift with respect to a solid sphere of the same size. This is entirely due to the electromagnetic properties of this geometry. A size dependent change of the dielectric properties will broaden the resonances and might shift the spectral position slightly, but it will not affect the general red-shift. The red-shift increases with decreasing thickness of the shell. This is the opposite behaviour to a homogeneous sphere where a red-shift is associated with an increase in particle size. 


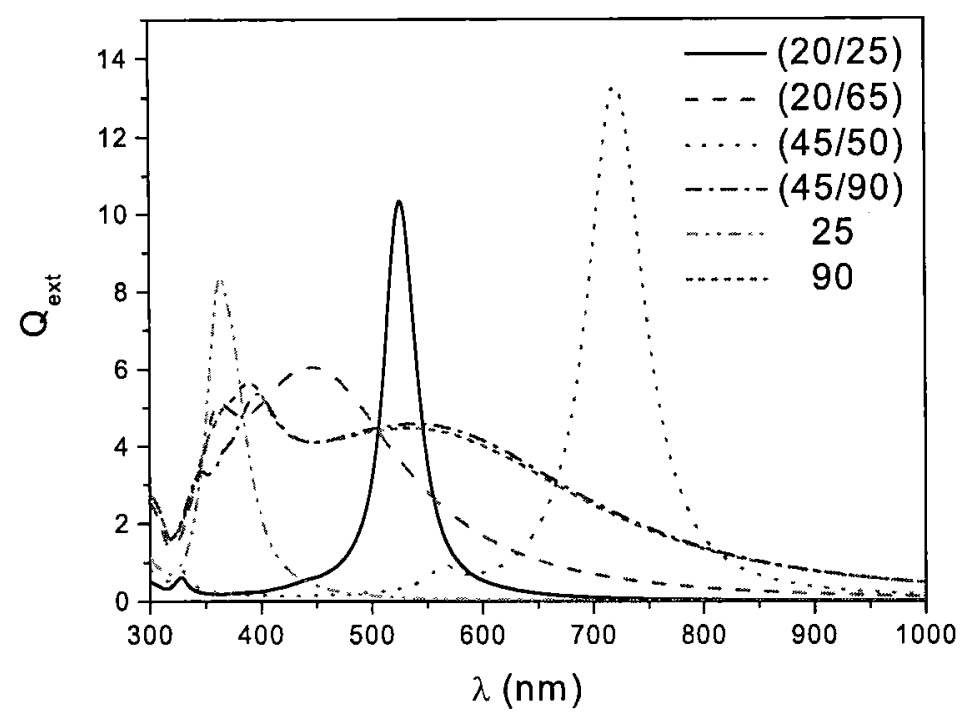

Figure 1. Extinction efficiency spectra for the spherical silver structures presented in this paper. The spectra for the shells are designated in the legend according to $\left(R_{\text {core }} / R_{\text {particle }}\right)(\mathrm{in} \mathrm{nm})$. The single number mean homogeneous spheres of the radius shown in the legend (in $\mathrm{nm}$ ).

The response of the shell can be explained in a rather simple way: A decrease in the shell thickness enhances the strength of the fields inside the shell. This is due to the fact that the field lines are focussed in a smaller region (the decreasing shell), which increases the field density and hence the field strength. It follows that the total dipole strength of the core-shell particle is increased and as this acts to compensate an external field, the compensation is enhanced and subsequently the energy of the LSPR reduces. This is the reason for the increased red-shift for thin metallic shells.

Despite the enhanced field inside the shell particle and at the surface, the immediate surroundings of the shell does not show this enhancement. A strong indication for this is that the cross sections of a clusters of shells are converging at a much faster rate than for a cluster of solid spheres. ${ }^{27}$ This is also and especially true for the touching case, where the difference in the number of expansion terms required can be as large as a factor of $30 .{ }^{27}$

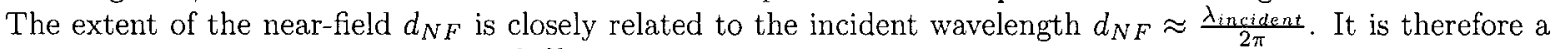
function of the size parameter $k a=\frac{2 \pi N a}{\lambda}$ of the sphere, where $N$ is the refractive index of the medium and $a$ is the radius of the sphere.

\section{RESULTS}

All the presented results are for $n_{\text {medium }}=1.0$ and a silica core with the respective refractive index at the studied wavelengths. Size effects are not taken into consideration in this paper. Figure 1 shows the spectra for the silver structures considered in this paper, namely shells with a silica core of radius $20 \mathrm{~nm}$ and $45 \mathrm{~nm}$ and shell thicknesses of 5,20 and $45 \mathrm{~nm}$. The spectra for homogeneous spheres with a radius of $25 \mathrm{~nm}$ and $45 \mathrm{~nm}$ are also included. What can be seen in this graph is the increasing red-shift with decreasing $\delta=\frac{\text { shell thickness }}{R_{\text {core }}}$ ratio. ${ }^{12}$ One can also notice that the extinction efficiency increases with decreasing $\delta$ ratio. This is mainly due to an enhanced absorption component for small $\delta$ values $(\lesssim 0.5)$ for not too large total sizes (for silver: $\left.R_{\text {total }} \lesssim 100 \mathrm{~nm}\right) .{ }^{28}$ The occurrence of quadrupole resonances in the larger particles can also be observed. For the $5 \mathrm{~nm}$ thick shell we can see that there is a high-energy resonance (at $\approx 330 \mathrm{~nm}$ ). This resonance is the 
a
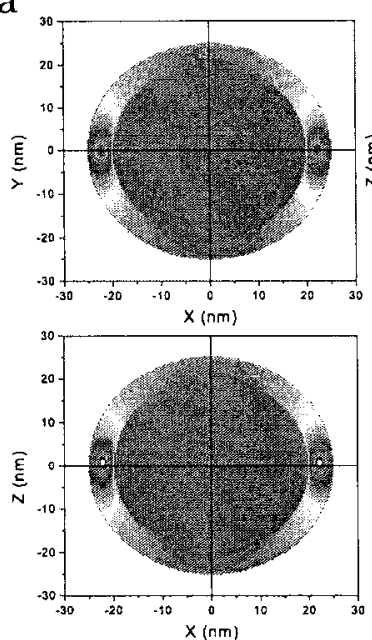
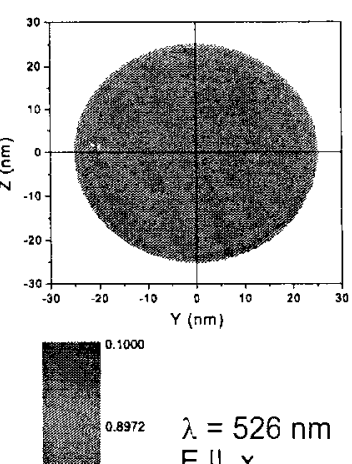

$E \| x$

$k \|+z$ b
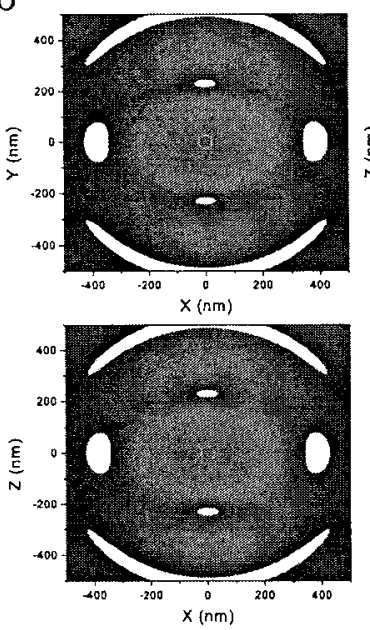
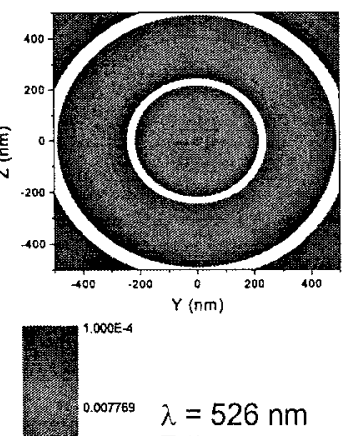

$E \| x$

$k \|+z$

Figure 2. (a) Internal and (b) external field strengths $|\mathbf{E}|^{2} /\left|\mathbf{E}_{0}\right|^{2}$ (logarithmic colour scale) for an $5 \mathrm{~nm}$ thick Ag shell on a silica core with $R_{\text {core }}=20 \mathrm{~nm}$ in the three principal cartesian coordinate planes (see the axes labels).

antisymmetric mode for the shell, which will be explained in more detail with Fig. 3. It is rather insensitive to the $\delta$ ratio. ${ }^{29}$

Figure 2 shows an overview of the field profile for a thin silver shell with $R_{\text {core }}=20 \mathrm{~nm}$ and $R_{\text {particle }}=25 \mathrm{~nm}$ at the dipole LSP resonance of $\lambda=526 \mathrm{~nm}$. The internal fields show a similar behaviour to that of metallic rings, ${ }^{29}$ which is distinctively different to that of a homogeneous sphere. The main difference is that the largest fields in the shell are perpendicular to the incident polarisation direction. In homogeneous spheres the largest fields are preferentially along the polarisation direction. This does not affect the external field pattern of the shells. If the shells are not too thick the field inside the core follows the incident field, i.e. the areas of large field strengths are oriented along the incident polarisation. This cannot been seen in Fig. 2 because of the small field gradient inside the core with respect to the one in the shell and hence the core looks homogeneous in this figure, especially with the logarithmic colour scale.

The external field shows the standard behaviour of the radiation pattern perpendicular to the incident polarisation direction, which is in our case along the $x$-axis and a rotationally symmetric pattern in the plane which is perpendicular to the incident polarisation. Differences between the $x, y$ and $x, z$ plane, which should be expected as the incident wave propagates along the positive $z$-axis, are not visible in this case, due to the small size of the particle. Because of this behaviour we will concentrate purely on results for the $x, z$-plane.

Another difference between a homogeneous sphere and a shell is that the shell exhibits two resonances, a low energy symmetric one and a high energy antisymmetric one. The field strength for the two cases and the according qualitative charge distributions are shown in Fig. 3. That the symmetric mode is the low energy one might be surprising as often symmetric modes are high energy solutions. The reason for this is that the symmetric mode indeed creates a higher energy solution for the particle. This means that the symmetric mode has the largest total dipole moment of the total particle. But as this moment acts to counterbalance the incident field, an increase in the moment means a decrease in the total energy of the system. Hence, it provides the lowest total energy solution. In the long wavelength or small particle limit one would expect that the response of the shell starts to become similar to that of a homogeneous particle, which means showing a behaviour closer to the high energy, antisymmetric response than the low energy, symmetric one. As a silver shell does not show this kind of behaviour for wavelengths of up to $3 \mu \mathrm{m}$ due to a large $n$, we show this case for a gold shell in Fig. 4 . As can be clearly seen in this far off-resonance case the internal field follows the incident polarisation direction, as in the homogeneous case.

Fig. 5 compares the external field for different core-shell systems. The numbers in brackets are showing the radii of the core and shell according to $\left(R_{\text {core }} / R_{\text {particle }}\right)$. The maximum range in each image is \pm 20 times the particle 

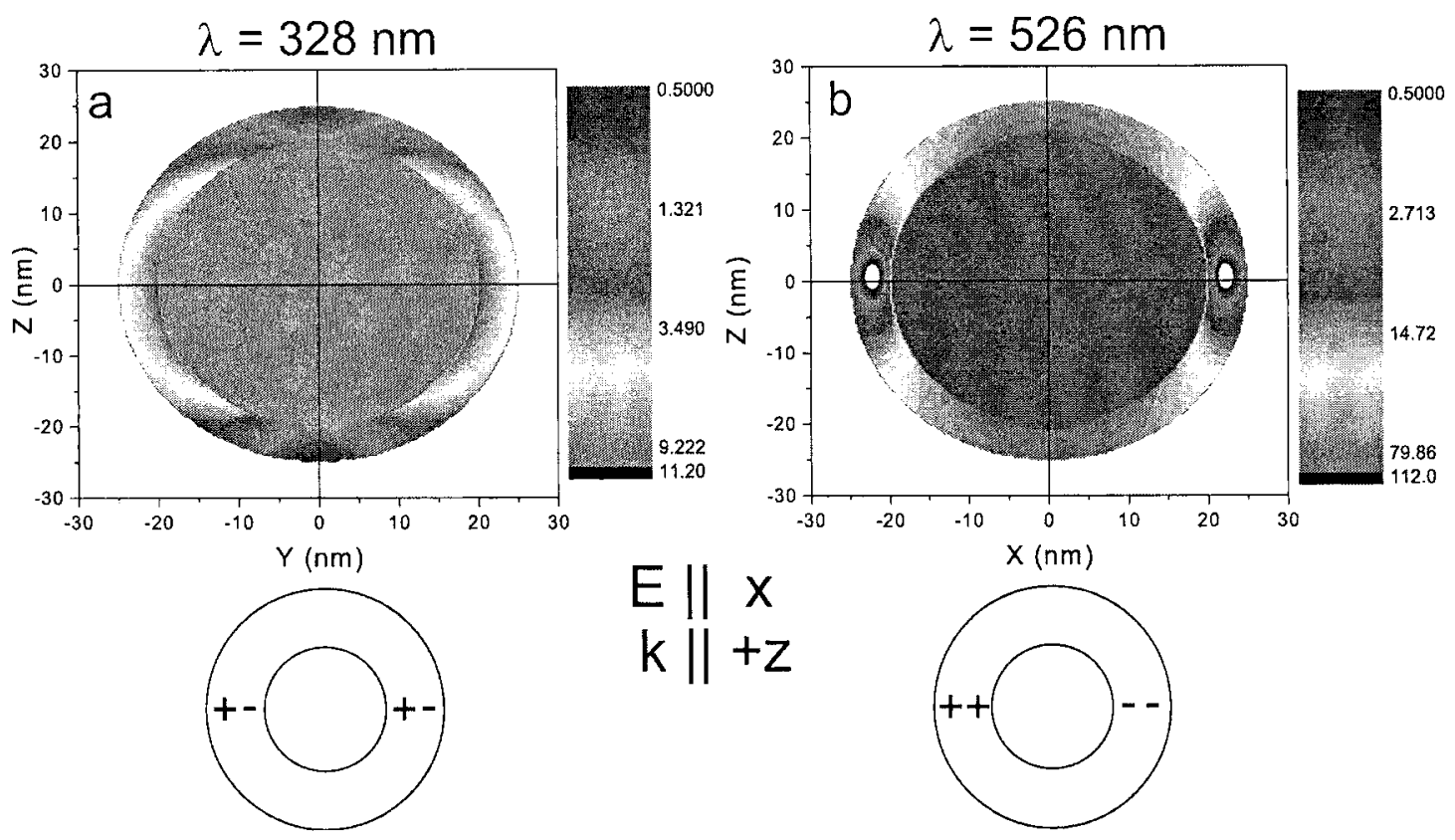

Figure 3. Comparison of the field strength $|\mathbf{E}|^{2} /\left|\mathbf{E}_{0}\right|^{2}$ (logarithmic colour scale) for the: (a) antisymmetric, high energy resonance and (b) symmetric, low energy resonance of a $5 \mathrm{~nm}$ thick $\mathrm{Ag}$ shell on a silica core with $R_{\text {core }}=20 \mathrm{~nm}$. The graphs below the field plots are showing the qualitative charge distribution for the two cases.

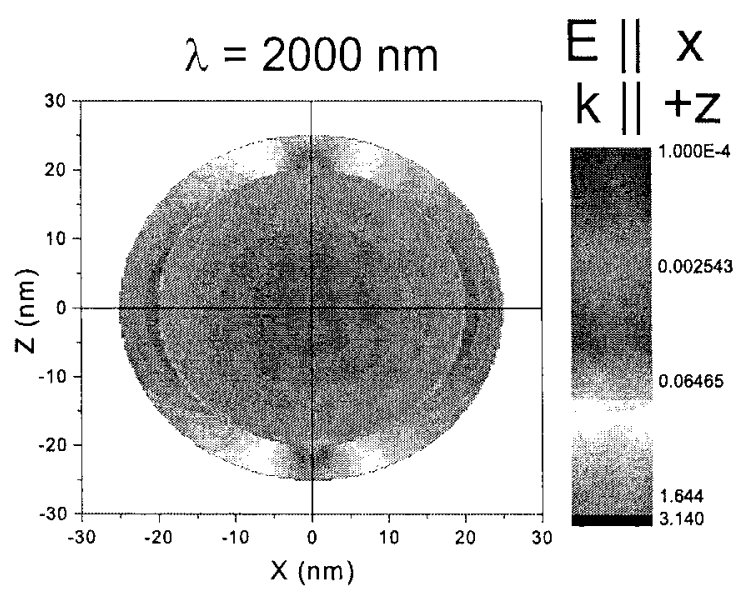

Figure 4. Field strength $|\mathrm{E}|^{2} /\left|\mathrm{E}_{0}\right|^{2}$ (logarithmic colour scale) for a gold shell with $R_{\text {core }}=20 \mathrm{~nm}$ and $R_{\text {particle }}=25 \mathrm{~nm}$ at the off-resonance wavelength of $2000 \mathrm{~nm}$, to show the response in the long wavelength, small particle limit. 

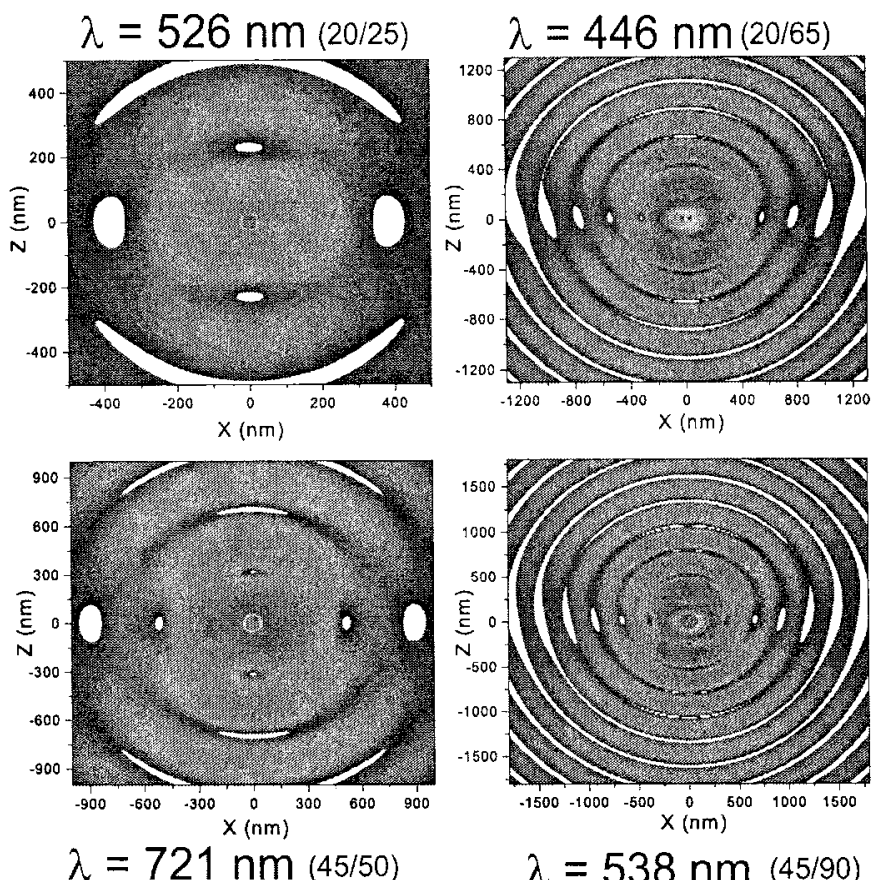

\section{$E \| x$}

$k \|+z$

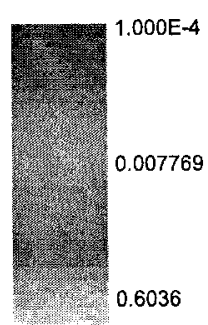

$\lambda=721 \mathrm{~nm}(45 / 50)$

Figure 5. Comparison of the external field strength $|\mathbf{E}|^{2} /\left|\mathbf{E}_{0}\right|^{2}$ (logarithmic colour scale) for different core-shell systems. The results are at the respective resonances, whose wavelengths are noted in the graph (the results for the $(20 / 65)$ and $(45 / 90)$ particle are from the dipole resonance). The numbers in brackets are corresponding to the radii of the core and shell according to $\left(R_{\text {core }} / R_{\text {particle }}\right)$. All images have a range of $\pm 20 R_{\text {particle }}$

radius, in order to enable direct comparison of the relative extent of the near-field. As can be seen the thinner the shell the larger the relative extent of the near-field, although the near-field intensity in the larger particle is larger. This is demonstration of the fact that the absorption of a metallic shell is inversely proportional to the shell-thickness to core-radius ratio. ${ }^{28}$ Because this ratio is lower for the larger particle it's absorption is higher. This means that more energy is stored directly inside the particle and the shell and hence at the surface of the shell. It is important to note that despite this and larger fields within the shell particle a homogeneous sphere still has a larger near-field intensity than a shell. The enhancement in the shell is only usable directly at the surface of the shell.

The difference in the extent of the near-field between shells and homogeneous particles can be seen in Fig. 6 . For the smaller particle of $R=25 \mathrm{~nm}$, the respective shell having a thickness of $5 \mathrm{~nm}$, the same contour line within the near-field of the solid sphere at resonance extends around $50 \mathrm{~nm}$ further from the particle than in the case of the shell. If the shell becomes thicker the differences between the field profiles of the shell and a solid sphere of the same size become negligible, because the shell will look to the incident wave more and more like a homogeneous sphere. The internal field profile for the quadrupole resonance of the larger particle $\left(R_{\text {core }}=45 \mathrm{~nm} / R_{\text {particle }}=90 \mathrm{~nm}\right)$ can be seen in Fig. 7 . The four poles are clearly distinguishable.

The differences in the near-field between a metallic shell and a solid metal particle also diminish at off-resonance wavelengths, up till the point where the responses are almost indistinguishable, because both particles fall then into the small particle (Rayleigh) limit with respect to that wavelength and the difference in the dielectric properties, due to the core, become negligible, especially for thicker shells. This result is further supported by the fact that particle interactions between metallic shells are not as strong as for homogeneous particles, which expresses itself in a faster convergence for close proximity clusters of shells. ${ }^{27}$ This remains true even for the notoriously difficult touching case. 
a
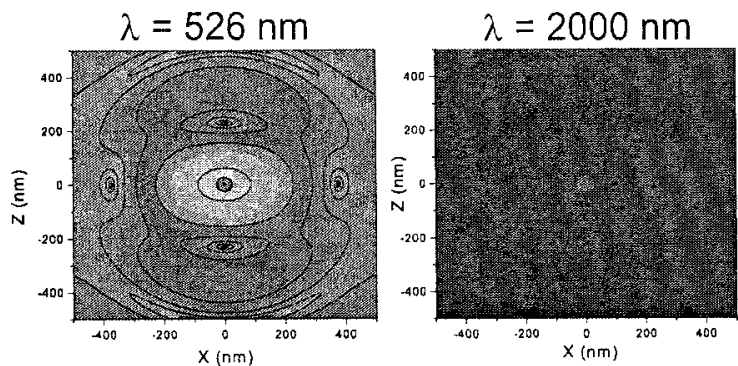

\section{$E \| x$}

$k \|+z$


b

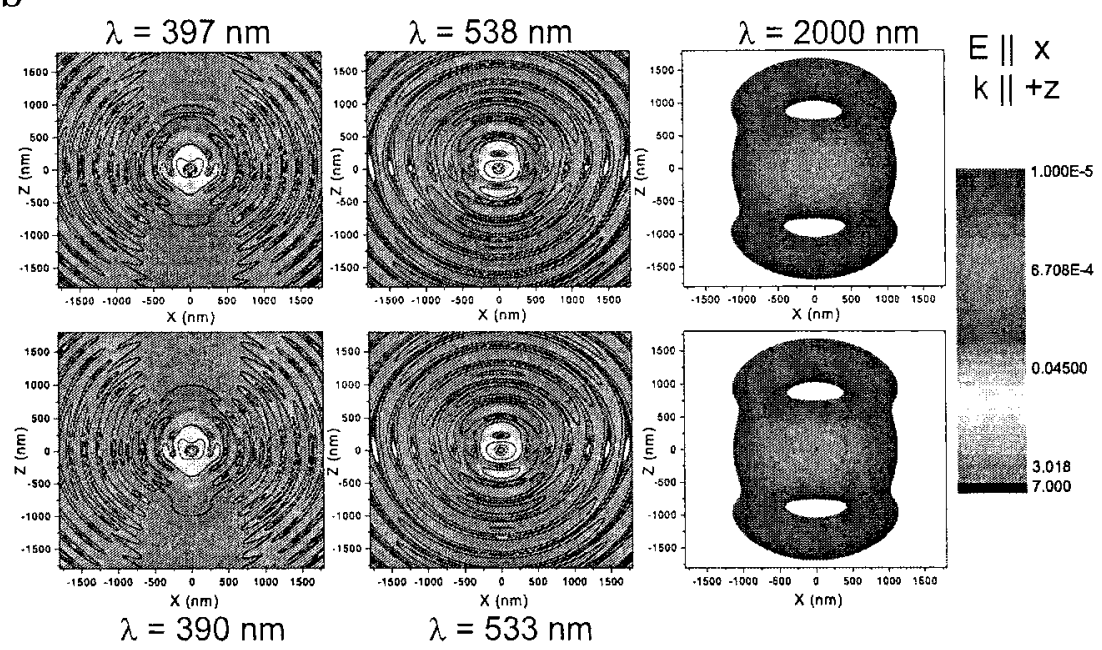

Figure 6. Comparison of $|\mathbf{E}|^{2} /\left|\mathbf{E}_{0}\right|^{2}$ between a spherical silver shell and and homogeneous silver sphere (logarithmic colour scale): (a) top row: shell $R_{\text {core }}=20 \mathrm{~nm} / R_{\text {particle }}=25 \mathrm{~nm}$; bottom row: sphere $R_{\text {sphere }}=25 \mathrm{~nm}$; the first column is at their respective dipole LSP resonance wavelength, as indicated in the figure and the second column is off-resonance at $2000 \mathrm{~nm}$. (b) top row: shell $R_{\text {core }}=45 \mathrm{~nm} / R_{\text {particle }}=90 \mathrm{~nm}$; bottom row: sphere $R_{\text {sphere }}=90 \mathrm{~nm}$; the first column is at their respective quadrupole, the second at their respective dipole LSPR wavelengths, as indicated in the figure and the third column is off-resonance at $2000 \mathrm{~nm}$. 


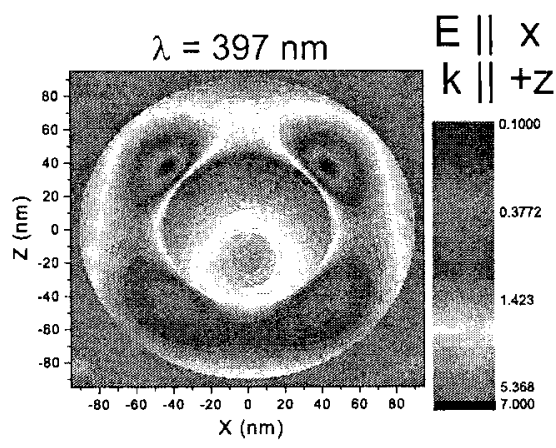

Figure 7. Internal field strength $|\mathbf{E}|^{2} /\left|\mathbf{E}_{0}\right|^{2} \quad$ (logarithmic colour scale) for the quadrupole resonance of a silver shell on a silica core with $R_{\text {core }}=45 \mathrm{~nm}$ and $R_{\text {particle }}=90 \mathrm{~nm}$.
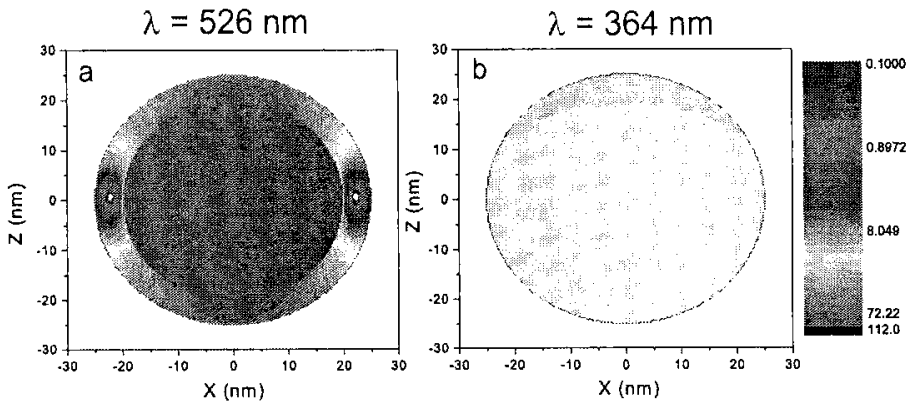

Figure 8. Comparison of the internal field strengths $|\mathrm{E}|^{2} /\left|\mathrm{E}_{0}\right|^{2}$ between a spherical silver shell and and homogeneous silver sphere at their respective resonances (logarithmic colour scale). (a) shell $R_{\text {core }}=$ $20 \mathrm{~nm} / R_{\text {particle }}=25 \mathrm{~nm}$; (b) sphere $R_{\text {sphere }}=25 \mathrm{~nm}$. (same as in Fig. $6(\mathrm{a}))$

This is further demonstrated in Fig. 8, which shows the internal fields for the same structures as in Fig. 6 a), namely a $R_{\text {core }}=20 \mathrm{~nm} / R_{\text {particle }}=25 \mathrm{~nm} \mathrm{Ag}$ shell and a $R_{\text {sphere }}=25 \mathrm{~nm} \mathrm{Ag}$ sphere. The internal and surface fields of the homogeneous sphere have a maximum value of $|\mathbf{E}|^{2} /\left|\mathbf{E}_{0}\right|^{2} \approx 20$, while the ones for the shell are stronger by roughly a factor of 5 . And while those large fields might be present at the surface of the shell and inside groves or between protrusions on the surface, they are much more confined to the actual surface than in the case of a homogenous particle. This is the only explanation why the response of shell clusters is faster converging than the one for solid sphere clusters (the difference in the number of terms can be as large as factor of 30 in the touching case). ${ }^{27}$

\section{CONCLUSION}

We showed the internal and external field strengths $|\mathbf{E}|^{2} /\left|\mathbf{E}_{0}\right|^{2}$ for silver metal shells on a dielectric core and discussed the influence of shell thickness and particle size. We also showed the response for homogeneous particles and the differences between these and the shell particles.

The profiles for the high energy, antisymmetric mode and the low energy, symmetric mode have been presented and discussed. One interesting point is that the field profile of the dominant low energy resonance shows large fields perpendicular to the incident polarisation direction, which might be a bit surprising. The reason why this symmetric case is the low energy mode is that it creates the largest total dipole (and multipole) moment for the whole particle and this counteracts the incident field. Hence, the symmetric case creates the lowest total energy resonance.

While thin shells are creating a larger field inside the particle, these fields do not extend far from the actual surface. In fact, they are much more confined to the particle surface than in the homogeneous case. This explains why calculations for clusters of shells are much faster converging compared to clusters of solid particles.

\section{ACKNOWLEDGMENTS}

S. Schelm acknowledges the support of his PhD by the BASF AG, Germany.

\section{REFERENCES}

1. J. B. Jackson, S. L. Westcott, L. R. Hirsch, J. L. West, and N. J. Halas, "Controlling the surface enhanced raman effect via the nanoshell geometry," Appl. Phys. Lett. 82(2), pp. 257-259, 2003.

2. C. Graf and A. van Blaaderen, "Metallodielectric colloidal core-shell particles for photonic applications," Langmuir 18(2), pp. 524-534, 2002. 
3. E. Hao, S. Y. Li, R. C. Bailey, S. L. Zou, G. C. Schatz, and J. T. Hupp, "Optical properties of metal nanoshells," J. Phys. Chem. B 108(4), pp. 1224-1229, 2004.

4. R. Baer, D. Neuhauser, and S. Weiss, "Enhanced absorption induced by a metallic nanoshell," Nano Letters 4(1), pp. 85-88, 2004.

5. S. L. Westcott, J. B. Jackson, C. Radloff, and N. J. Halas, "Relative contributions to the plasmon line shape of metal nanoshells," Phys. Rev. B 66(15), 2002.

6. G. Mie, "Beiträge zur optik trüber medien, speziell kolloidaler metalllösungen," Ann. Phys. 25(4), pp. 377$445,1908$.

7. A. Aden and M. Kerker, "Scattering of electromagnetic waves from two concentric spheres," J. Appl. Phys. 22, pp. 1242-1246, 1951.

8. A. Güttler Ann. Phys. 11, p. 65, 1952.

9. H. S. Zhou, I. Honma, H. Komiyama, and J. W. Haus, "Controlled synthesis and quantum-size effect in gold-coated nanoparticles," Phys. Rev. B 50(16), pp. 12052-12056, 1994.

10. R. D. Averitt, D. Sarkar, and N. J. Halas, "Plasmon resonance shifts of au-coated au2s nanoshells: Insight into multicomponent nanoparticle growth," Phys. Rev. Lett. 78(22), pp. 4217-4220, 1997.

11. A. J. Haes, S. L. Zou, G. C. Schatz, and R. P. Van Duyne, "A nanoscale optical biosensor: The long range distance dependence of the localized surface plasmon resonance of noble metal nanoparticles," J. Phys. Chem. B 108(1), pp. 109-116, 2004.

12. S. J. Oldenburg, R. D. Averitt, S. L. Westcott, and N. J. Halas, "Nanoengineering of optical resonances," Chem. Phys. Lett. 288(2-4), pp. 243-247, 1998

13. S. J. Oldenburg, S. L. Westcott, R. D. Averitt, and N. J. Halas, "Surface enhanced raman scattering in the near infrared using metal nanoshell substrates," J. Chem. Phys. 111(10), pp. 4729-4735, 1999.

14. Z. J. Jiang and C. Y. Liu, "Seed-mediated growth technique for the preparation of a silver nanoshell on a silica sphere," J. Phys. Chem. B 107(45), pp. 12411-12415, 2003.

15. Y. G. Sun and Y. N. Xia, "Increased sensitivity of surface plasmon resonance of gold nanoshells compared to that of gold solid colloids in response to environmental changes," Analytical Chemistry 74(20), pp. 5297$5305,2002$.

16. E. Prodan and P. Nordlander, "Electronic structure and polarizability of metallic nanoshells," Chem. Phys. Lett. 352(3-4), pp. 140-146, 2002.

17. C. Oubre and P. J. Nordlander, "Finite difference time-domain studies of optical properties of nanoshell structures," in Proceedings of SPIE Vol.5221 Plasmonics: Metallic Nanostructures and Their Optical Properties, N. J. Halas, ed., pp. 133-143, (SPIE, Bellingham, WA, 2003).

18. O. B. Toon and T. P. Ackerman, "Algorithms for the calculation of scattering by stratified spheres," Appl. Opt. 20(20), pp. 3657-3660, 1981.

19. C. F. Bohren and D. R. Huffman, Absorption and Scattering of Light by Small Particles, Wiley Science Paperback Series, Wiley-Interscience, 1998.

20. T. Kaiser and G. Schweiger, "Stable algorithm for the computation of mie coefficients for scattered and transmitted fields of a coated sphere," Computers in Physics 7(6), pp. 682-686, 1993.

21. For a comprehensive (not necessarily complete) list of programs refer to: http://atol.ucsd.edu/ p platau/scatlib/.

22. W. Yang, "Improved recursive algorithm for light scattering by a multilayered sphere," Appl. Opt. 42(9), pp. $1710-1720,2003$.

23. T. Kaiser, S. Lange, and G. Schweiger, "Structural resonances in a coated sphere - investigation of the volume-averaged source function and resonance positions," Appl. Opt. 33(33), pp. 7789-7797, 1994.

24. G. Roll, T. Kaiser, and G. Schweiger, "Eigenmodes of spherical dielectric cavities: coupling of internal and external rays," J. Opt. Soc. Am. A 16(4), pp. 882-895, 1999.

25. L. Kai and A. Dalessio, "Internal-field characteristics of spherical particles," Part. Part. Syst. Charact. 12(5), pp. 237-241, 1995.

26. R. Fenn and H. Oser, "Scattering properties of concentric soot-water spheres for visible and infrared light," Appl. Opt. 4, pp. 1504-1509, 1965. 
27. G. B. Smith and V. N. Pustovit, "Coupled multipolar interactions in clusters of nanoparticles with metal shells," Opt. Commun. 211(1-6), pp. 197-204, 2002.

28. S. Schelm and $\mathrm{G}$. Smith to be published.

29. J. Aizpurua, P. Hanarp, D. S. Sutherland, M. Kall, G. W. Bryant, and F. J. Garcia de Abajo, "Optical properties of gold nanorings," Phys. Rev. Lett. 90(5), p. 057401, 2003. 